Schumacher, Susanne; Stadler-Altmann, Ulrike; Emili, Enrico A.

\title{
Piktogramme als Unterstützungsmedien. Studien im Kindergarten zu Effekten von Bilderbüchern mit zusätzlichen Symbolen
}

Holub, Barbara [Hrsg.]; Himpsl-Gutermann, Klaus [Hrsg.]; Mittlböck, Katharina [Hrsg.]; Musilek-Hofer, Monika [Hrsg.]; Varelija-Gerber, Andrea [Hrsg.]; Grünberger, Nina [Hrsg.]: lern.medien.werk.statt. Hochschullernwerkstätten in der Digitalität. Bad Heilbrunn : Verlag Julius Klinkhardt 2021, S. 248-270. - (Lernen und Studieren in Lernwerkstätten)

Quellenangabe/ Reference:

Schumacher, Susanne; Stadler-Altmann, Ulrike; Emili, Enrico A.: Piktogramme als

Unterstützungsmedien. Studien im Kindergarten zu Effekten von Bilderbüchern mit zusätzlichen Symbolen - In: Holub, Barbara [Hrsg.]; HimpsI-Gutermann, Klaus [Hrsg.]; Mittlböck, Katharina [Hrsg.]; Musilek-Hofer, Monika [Hrsg.]; Varelija-Gerber, Andrea [Hrsg.]; Grünberger, Nina [Hrsg.]: lern.medien.werk.statt. Hochschullernwerkstätten in der Digitalität. Bad Heilbrunn : Verlag Julius Klinkhardt 2021, S. 248-270 - URN: urn:nbn:de:0111-pedocs-228247 - DOI: 10.25656/01:22824

https://nbn-resolving.org/urn:nbn:de:0111-pedocs-228247

https://doi.org/10.25656/01:22824

in Kooperation mit / in cooperation with:

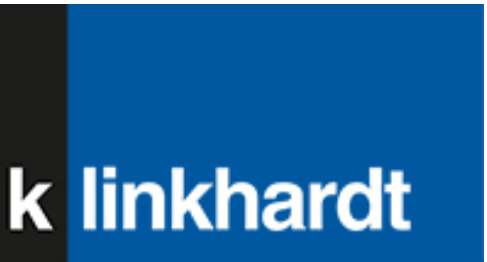

http://www.klinkhardt.de

\section{Nutzungsbedingungen}

Dieses Dokument steht unter folgender Creative Commons-Lizenz: http://creativecommons.org/licenses/by-nc-sa/4.0/deed.de - Sie dürfen das Werk bzw. den Inhalt unter folgenden Bedingungen vervielfältigen, verbreiten und öffentlich zugänglich machen sowie Abwandlungen und Bearbeitungen des Werkes bzw. Inhaltes anfertigen: Sie müssen den Namen des Autors/Rechteinhabers in der von ihm festgelegten Weise nennen. Dieses Werk bzw. der Inhalt darf nicht für kommerzielle Zwecke verwendet werden. Die neu entstandenen Werke bzw. Inhalte dürfen nur unter Verwendung von Lizenzbedingungen weitergegeben werden, die mit denen dieses Lizenzbedingungen weitergegeben werden,

Mit der Verwendung dieses Dokuments erkennen Sie die Nutzungsbedingungen an.

\section{Terms of use}

This document is published under following Creative Commons-License: http://creativecommons.org/licenses/by-nc-sa/4.0/deed.en - You may copy, distribute and transmit, adapt or exhibit the work in the public and alter, transform or change this work as long as you attribute the work in the manner specified by the author or licensor. You are not allowed to make commercial use of the work. If you alter, transform, or change this work in any way, you may distribute the resulting work only under this or a comparable license.

By using this particular document, you accept the above-stated conditions of

\section{Kontakt / Contact:}

\section{DeDOCS}

DIPF | Leibniz-Institut für Bildungsforschung und Bildungsinformation Informationszentrum (IZ) Bildung

E-Mail: pedocs@dipf.de

Internet: www.pedocs.de

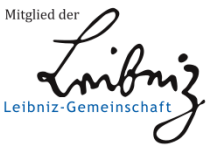




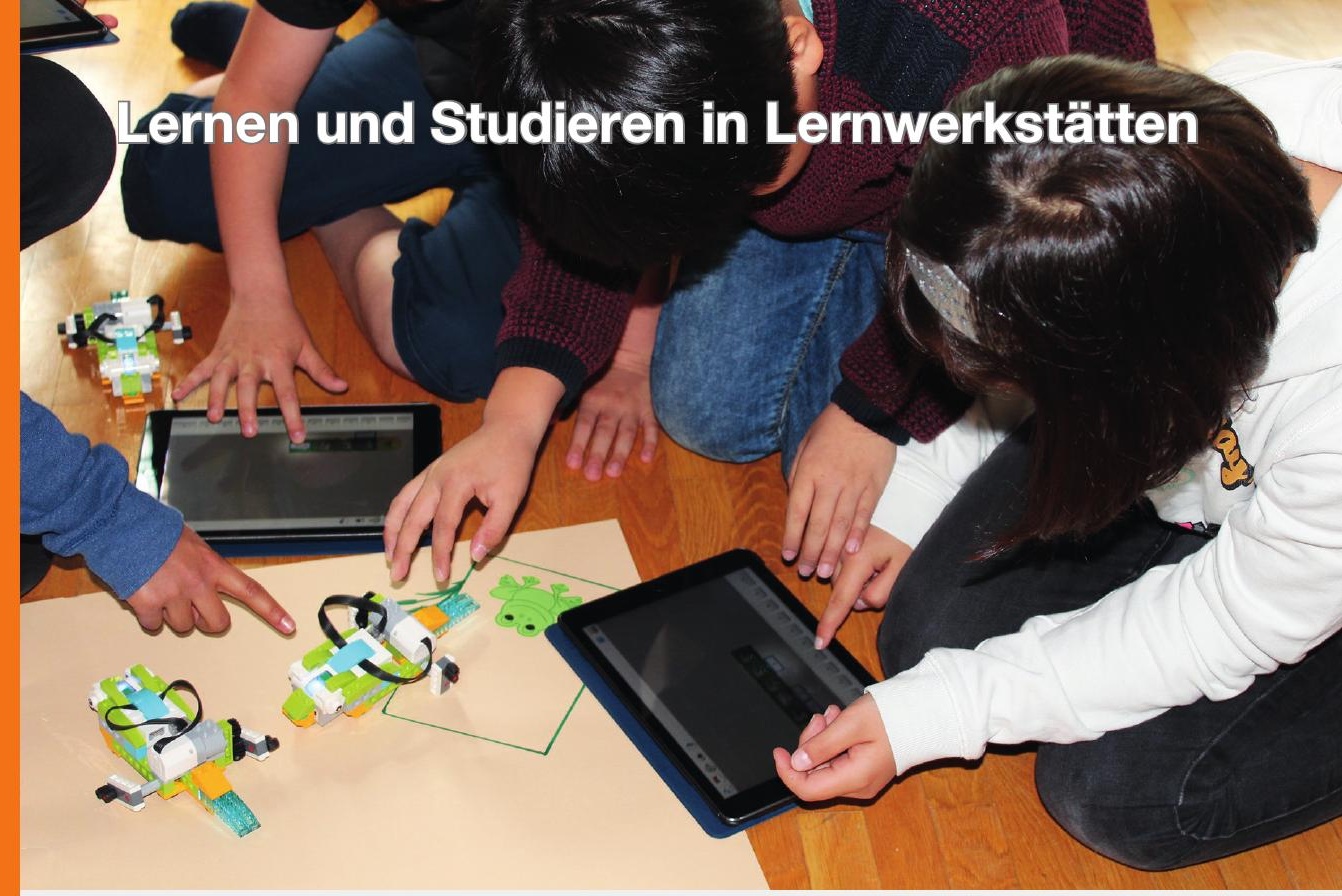

Barbara Holub / Klaus Himpsl-Gutermann Katharina Mittlböck / Monika Musilek-Hofer Andrea Varelija-Gerber / Nina Grünberger (Hrsg.)

\section{lern.medien.werk.statt}

Hochschullernwerkstätten in der Digitalität 
Barbara Holub Klaus Himpsl-Gutermann Katharina Mittlböck Monika Musilek-Hofer Andrea Varelija-Gerber

Nina Grünberger (Hrsg.)

\section{lern.medien.werk.statt}

Hochschullernwerkstätten in der Digitalität 
Dieser Titel wurde in das Programm des Verlages mittels eines Peer-Review-Verfahrens aufgenommen. Für weitere Informationen siehe www.klinkhardt.de.

Bibliografische Information der Deutschen Nationalbibliothek Die Deutsche Nationalbibliothek verzeichnet diese Publikation in der Deutschen Nationalbibliografie; detaillierte bibliografische Daten sind im Internet abrufbar über http://dnb.d-nb.de.

2021.ig. (C) by Julius Klinkhardt.

Coverfoto: (c) ZLI PH Wien.

Druck und Bindung: AZ Druck und Datentechnik, Kempten.

Printed in Germany 2021.

Gedruckt auf chlorfrei gebleichtem alterungsbeständigem Papier.

Die Publikation (mit Ausnahme aller Fotos, Grafiken und Abbildungen) ist veröffentlicht unter der Creative Commons-Lizenz: CC BY-NC-SA 4.0 International https://creativecommons.org/licenses/by-nc-sa/4.0/

ISBN 978-3-7815-5904-2 digital doi.org/10.35468/5904

ISBN 978-3-7815-2468-2 print 


\section{Inhaltsverzeichnis}

Barbara Holub, Klaus Himpsl-Gutermann, Katharina Mittlböck, Monika Musilek-Hofer, Andrea Varelija-Gerber und Nina Grünberger

Einführung in den Band 11

\section{Zur Konstituierung von Hochschullernwerkstätten}

Sandra Tänzer

Mut - Zumutung - Ermutigung. Oder wie man lernt, eine Hochschullernwerkstatt zu lieben

Markus Peschel, Hartmut Wedekind, Pascal Kihm und Mareike Kelkel

Hochschullernwerkstätten und Lernwerkstätten -

Verortung in didaktischen Diskursen

Dietlinde Rumpf und Corinna Schmude

Von der Herausforderung, die Vielfalt von Hochschullernwerkstätten

in einer Definition abzubilden

Pascal Kihm und Markus Peschel

„Komplexität wagen!“ - Methoden zur Beforschung von

offenen Lehr-Lern-Prozessen in Hochschullernwerkstätten 70

\section{Zum Spannungsverhältnis von Hochschullernwerkstätten und Digitalität}

Mark Weißhaupt, Ralf Schneider, Clemens Griesel und Agnes Pfrang

Digitale Erfahrung? Über das Lernen zwischen Instruktion und

(Ko-)Konstruktion

Stefan Brée, Lena S. Kaiser und Tanja Wittenberg

Lernwerkstatt als digitaler Erfahrungsort. Potenziale und

Herausforderungen für Lernwerkstätten als Orte

„offener multimedialer Produktionsästhetik“

\section{Ulrike Stadler-Altmann und Gerda Winkler}

Real \& virtuell, analog \& digital: Dimensionen einer Kooperation.

Multifunktionalität als Kennzeichen zukunftsfähiger Lernwerkstatt- und

Bibliothekskonzeption 


\section{Josef Buchner und Michael Kerres}

Lernwerkstattarbeit in der digital vernetzten Welt.

Die Perspektive der gestaltungsorientierten Mediendidaktik

Sabrina Tietjen und Silvia Thünemann

Forschungswerkstatt digital: ein modernes Lehr-Lernarrangement

für eine Digitalisierungsstrategie im Lehramt?

\section{Zu fach- und mediendidaktischen Perspektiven}

Johannes Mayer, Antonia Lemensieck, Maria Reinhardt und Karl Wollmann

Fachliche Perspektiven auf digitalisierungsbezogene Lernangebote

in der Ausbildung von Grundschullehrer*innen

Heike Hagelgans

Die Thematisierung digitaler Medien in den schulpraktischen Studien

Fachdidaktische Reflexionen von digitalen Medien für das Lernen im

Mathematikunterricht der Primarstufe

Jeanette Hoffmann, Katharina Egerer und Franziska Herrmann

Analoge Bilder - digitaler Film.

Möglichkeitsräume für literarästhetisches Lernen in

Hochschullernwerkstätten

Michael Rieseneder und Wolfgang Wagner

Erstes Programmieren mit Kindern über Handlungserfahrungen.

Das Konzept Activity-based-Coding

Patrick Isele und Julia Höke

Reflexionen über digitales Lernen mit dem Sphero SPRK+

Erfahrungen mit Studierenden in der „Lernwerkstatt ${ }^{3 “}$

Susanne Schumacher, Ulrike Stadler-Altmann und Enrico A. Emili

Piktogramme als Unterstützungsmedien.

Studien zu Effekten von Bilderbüchern mit zusätzlichen Symbolen im Kindergarten 


\section{Zu Aspekten, Perspektiven und Herausforderungen von Hochschullernwerkstätten}

Sabine Fischer und Max de Baey-Ernsten

Didaktische Perspektiven einer ästhetischen Werkstatt

Tanja Wittenberg und Lena S. Kaiser

„Ich war frustriert, ich bin kein Kind mehr“ - Erfahrungslernprozesse

mit verwendungs- und bedeutungsoffenem Material in

kindheitspädagogischen Hochschullernwerkstätten

Lisa Eßel und Laura Schlichting

Hochschullernwerkstätten im Spannungsfeld zwischen

Freiwilligkeit und Pflicht

Mareike Kelkel, Markus Peschel und Pascal Kihm

Potenziale der pädagogisch-didaktischen Öffnung in

Hochschullernwerkstätten

Barbara Holub und Sybille Roszner

Hochschullernwerkstatt - Ausgangspunkt für

persönlichkeitsorientierte Professionalisierung in der Ausbildung

für Lehrpersonen

Verzeichnis der Autor*innen 


\author{
Susanne Schumacher, Ulrike Stadler-Altmann und \\ Enrico A. Emili
}

\title{
Piktogramme ${ }^{1}$ als Unterstützungsmedien Studien im Kindergarten zu Effekten von Bilderbüchern mit zusätzlichen Symbolen
}

\begin{abstract}
Zentrale Aufgabe der EduSpace-Lernwerkstatt der Freien Universität Bozen (unibz) am Standort Brixen ist die Professionalisierung von pädagogischen Fachkräften im Kindergarten und Lehrpersonen in der Schule (Stadler-Altmann, 2018; Schumacher et al., 2020). Dies geschieht im Rahmen des Auftrags der Hochschule teils im Masterstudiengang für den Primarbereich, teils im postgradualen Austausch zwischen Wissenschaft und Praxis.

Im Interesse des - Südtiroler - Kindergartens liegt es, pädagogische Fachkräfte für den "Umgang mit individuellen Unterschieden und Inklusion zu sensibilisieren" (RRL, 2008, S. 20ff.) sowie im Bildungsfeld „kommunikationsfreudige und medienkompetente Kinder" (RRL, 2008, S. 32ff.) fortzubilden. Dieses Interesse wird in den Angeboten der EduSpace-Lernwerkstatt aufgegriffen und fießt in entsprechende Forschungsaktivitäten ein.

Um Kindern mit besonderen Bildungsbedürfnissen (BES) die Möglichkeit der Weltaneignung und Kommunikation zu erleichtern, bedarf es entsprechender Maßnahmen (UN, 2006), wie etwa den Einsatz von Augmentative and Alternative Communication (AAC) bzw. Unterstützter Kommunikation (UK).

Dieser Beitrag konzentriert sich auf die Umwandlung von in Südtiroler Kindergärten verwendeten Kinder- und Bilderbüchern in Bücher mit zusätzlichen Symbolen ${ }^{2}$ nach dem INbook-Modell (csinbook, 2017). Im Mittelpunkt stehen dabei der Kompetenzaufbau der pädagogischen Fachkräfte im Bereich der Inklusion und der Unterstützten Kommunikation sowie der Transformationsprozess von textbasierten Inhalten im bimodalen, nicht-sprachlichen Repräsentationssystem. Die erzielten Forschungsergebnis-
\end{abstract}

1 Piktogramme sind einfache, auf das Wesentliche reduzierte Zeichen mit Aufforderungscharakter (Böhringer, 2011, S. 433).

2 Der sprachwissenschaftlich treffendere Begriff wäre grafisches Zeichen, um eine deutlichere Abgrenzung zur Lautsprache vorzunehmen (vgl. Prosetzky, 2002, S. 22). 
se werden den Qualitätsebenen der Intervention nach Giel (1999) zugeordnet und abschließend in Bezug auf den Theorie-Wissens-Transfer in einer Hochschullernwerkstatt diskutiert.

\section{Professionalisierung von pädagogischen Fachkräften und Lehrkräften}

Der bildungswissenschaftliche Masterstudiengang für den Primarbereich an der Freien Universität Bozen (unibz) führt in fünf Jahren zu einem berufsqualifizierenden Abschluss. Absolvent*innen erhalten mit dem bestandenen Abschluss die Befähigung für die pädagogische Arbeit als Kindergärtner*in oder Lehrkraft in den jeweiligen Bildungseinrichtungen sowie die Lehrbefähigung für den Englischunterricht an der Grundschule. Organisiert ist das Südtiroler Bildungssystem nach den nationalen italienischen Vorgaben. Gelehrt und gelernt wird getrennt nach den drei Landessprachen: Deutsch, Italienisch und Ladinisch (siehe Legislativdekret vom 24. Juli 1996, Nr. 434), sowohl in den Kindergärten und Schulen als auch an der Universität.

Innerhalb der akademischen Ausbildung verläuft der Wissenstransfer in Feedbackschleifen, in dem sich Wissenschaftler*innen wie auch Praktikumskoordinator*innen und Studierende als forschend Lernende verstehen, die ihre Überlegungen in arbeitsteiliger Interaktion austauschen. Den regionalen Transfer wissenschaftlicher Erkenntnisse unterstützt die unibz durch die Öffnung von Fachtagungen u.a. für interessierte pädagogische Fachkräfte im Kindergarten und Lehrpersonen in der Grundschule, Kooperationen mit der Bildungsdirektion sowie den Schul- und Kindergartensprengeln. Dieser Wissenstransfer wird in erster Linie durch die EduSpace-Lernwerkstatt gewährleistet (Stadler-Altmann et al., 2020).

\subsection{Didaktische Ideen und Anregungen aus der EduSpace-Lernwerkstatt}

Hochschullernwerkstätten können mit Blick auf den Theorie-Praxis-Transfer und das Lebenslange Lernen im Kontext der kontinuierlichen pädagogischen Professionalisierung zentrale Aufgaben und Funktionen übernehmen (vgl. Bubolz-Lutz \& Mörchen, 2011). Ausgehend vom inhaltlichen Konzept eines konstruktionsorientierten und konstruktivistischen Ansatzes (Stadler-Altmann, 2018) dient die EduSpace-Lernwerkstatt nicht nur als Lernumgebung zur Vermittlung von berufspraktischem Faktenwissen (Stadler-Altmann, 2019) oder zum Einüben von prozeduralem Anwendungswissen (Schumacher et al., 2019), sondern auch als Raum zur Reflexion von didaktischen und methodischen Praxiserfahrungen durch Forschendes Lernen (Huber, 2009; Wedekind, 2013). 
Das Konzept der inklusiven Bildung im deutschsprachigen Kindergarten betont den Wert der Vielfalt menschlicher Ausdrucksformen (RRL, 2008, S. 17). So finden sich in den Lerngemeinschaften Kinder unterschiedlichen Alters, mit unterschiedlichem sozialem Hintergrund, aus anderen Sprach- und Kulturkreisen und Kinder mit besonderen Bildungsbedürfnissen (BES). Den pädagogischen Fachkräften kommt unter anderem die Aufgabe zu, die sprachlichen Kompetenzen der Kinder in vielfältigen Lebenssituationen zu fördern. Die gemeinsame Bilderbuchbetrachtung stellt dabei nicht nur einen ersten Zugang zum Schriftspracherwerb und für die Leseentwicklung dar, sondern ist vielmehr der Schlüssel zur Auseinandersetzung mit unterschiedlichen Lebensweisen und gesellschaftlichen Normen (Keunecke, 2000). Die professionelle Begleitung verlangt von den pädagogischen Fachkräften deshalb neben linguistischem Fachwissen auch entwicklungspsychologische Kenntnisse u.a. zur Konstitution geschlechtlicher Zugehörigkeit und der damit verbundenen Identitätsbildung von Kindern, Diagnosekompetenzen für die Sprachstanderhebung und angemessene didaktisch-methodische Kompetenzen, um den Prozess der Sprachaneignung unterstützen zu können.

\subsection{Bedeutungserwerb in Lautsprache und Unterstützter Kommunikation}

Der Bedeutungserwerb rein sprachlicher wie auch visueller Zeichen erfolgt nach den gleichen semiotischen und semantischen Regeln. Für beide gilt, dass ein zweidimensionales grafisches Zeichen durch die Zuschreibung einer bestimmten Bedeutung zum Symbol wird. Erst wenn die Verknüpfung dauerhaft hergestellt ist, wird Kommunikation möglich. Die besondere Herausforderung für pädagogische Fachkräfte bei der multimodalen Erweiterung eines bereits ausformulierten Bilderbuchtexts wie auch für Kinder bei der Bilderbuchbetrachtung besteht darin, zum jeweiligen schriftsprachlichen Begriff zusätzlich ein visuelles Zeichen zu erlernen (Hallbauer \& Kritzinger, 2016, S. 1).

Linguistisch gesehen werden sprachbasierte Informationen auf der textbasierten Repräsentationsebene mit Bildinformationen auf einer nicht-textbasierten Ebene verknüpft. Repräsentation beschreibt eine mental konstruierte Entität, die in einer kausalen Beziehung zu einer zweiten externen realen Entität steht. Zwischen den Ebenen werden zudem propositionale Einheiten gebildet, die als Bedeutungseinheiten auf dritter Ebene an das Vorwissen der*des Lesenden anknüpfen und bestenfalls die strukturelle Fähigkeit ausbilden, Situationsmodelle (visuelle Vorstellung ohne reale Anschauung) aufzubauen.
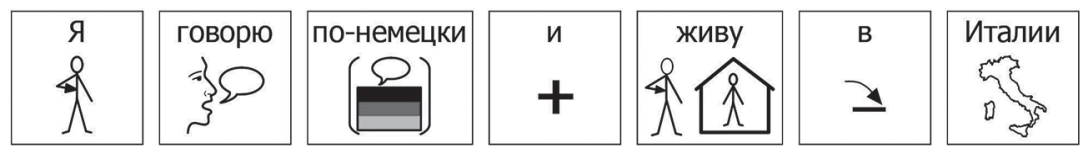

Widgit Symbols @ Widgit Software 2002-2020 - www.widgit.com

Abb. 1: Verknüpfung von sprachbasierten Informationen mit Bildinformationen 
Eine erfolgreiche Modellkonstruktion überschreitet die Optionen und das Handeln in einer bekannten Anforderungssituation und kann als Erweiterung der Kommunikationsfähigkeit bezeichnet werden. In den Umgang mit Repräsentationen können antizipierte Intentionen, Wünsche und Erwartungen von anderen Personen einfließen und zu einer Akkommodation in der Nutzung des externen Repräsentationssystems führen. Bereits Vorschulkinder sind fähig, alle drei Ebenen des Textverstehens herzustellen (Nieding, 2006).

Die in der barrierefreien Kommunikation (Maaß \& Rink, 2018) beschriebene Verarbeitung von Information ist sequenziell-analytisch gedacht. Es wird davon ausgegangen, dass in Wörtern der Sprache (Signifikate) die Bedeutung von Begriffen kodifiziert ist (Signifikante) und diese Bedeutung mit der Sprache abgerufen und rekonstruiert bzw. interpretiert werden kann. Dieser Gebrauch ist seinerseits wieder abhängig von den Personen mit ihrer jeweils individuellen Prägung durch einen soziokulturellen Hintergrund sowie vom aktuellen Verwendungskontext. Empirisch dokumentieren lassen sich diese Vorgänge durch Beobachtung des Umgangs mit gegebenen Informationen und der dadurch bedingten Aktivierung begrifflicher Assoziationen. Werden Begriffe wiederholt semantisch ähnlich benutzt, handelt es sich um semantisches Priming. Die Bahnungseffekte zeichnen sich durch geringe Reaktionszeit und geringe Fehlerzahl aus. Verweist der benutzte Begriff auf ein ähnliches Phänomen bzw. einen verwandten Sachverhalt in der gemeinsam erlebten Realität, liegt eine Verknüpfung mit dem gemeinschaftlichen Bedeutungsraum vor, die als semantisches Grounding bezeichnet werden kann (Harnad, 1990). Konstruktivistische Modelle betonen, dass die Bedeutung von Information nicht ohne den Gebrauch von Begriffen entwickelt werden kann (Gödert, 2018).

In der frühen Kindheit kommen Bilderbücher als erste externe Repräsentationsmedien zum Einsatz, die vor allem den visuellen Sinneseindruck ansprechen. Für Kleinkinder wird zudem oftmals der taktile Sinn einbezogen, indem das Fell eines Haustiers auch haptisch simuliert wahrnehmbar ist oder die Textur eines Kleidungsstücks für das Kind spürbar wird. Auditiv ist das Kind zu erreichen, indem Geschichten vorgelesen werden oder eine Gesprächssituation über das Gesehene geschaffen wird. Über etwas zu sprechen bedeutet, dass durch das Wahrnehmen von Umwelterfahrungen (Bilderbuch anschauen) und Vergegenwärtigen von Gedächtnisleistungen (an die Geschichte erinnern) sprachbasiert objektiviert und externalisiert werden kann. Der Verarbeitungsprozess beinhaltet aus Sicht der pädagogischen Psychologie dabei die Konfiguration kohärenter und konsistenter Schemata bzw. mentaler Repräsentationen (Schnotz, 2019, S. 99). Diese können bei Bedarf zu einem späteren Zeitpunkt rekonstruiert bzw. reaktiviert werden.

Neben der sinnlich-ästhetischen Wahrnehmung und Wahrnehmungsverarbeitung sprachbasierter Interaktion spielt die Sozialität beim Affiziertwerden eine Rolle (Knoblauch, 2017, S. 134). Die sozial- und kommunikationstheoretischen 
Diskursergebnisse spiegeln sich in den Handreichungen zum Bildungsfeld Sprache, Schriftsprachkultur, Zwei- und Mehrsprachigkeit (2008) und Best-PracticeAnleitungen (WIR, 2020, S. 7) der deutschsprachigen Kindergärten in Südtirol wider.

\subsection{Qualitätsmerkmale der Kommunikation mittels IN-books}

Der internationalen Kodifizierung von Menschenrechten zufolge stellt der Zugang zu Information eine wichtige Voraussetzung für eine funktionierende Demokratie dar und ist somit u.a. auf politische, kulturelle und soziale Teilhabe bzw. Interaktion im gesellschaftlichen Kontext ausgerichtet (AEMR, 2019, S. 19). Artikel 21 der UN-BKR führt näher dazu aus, dass Teilhabende im Rahmen einer kommunikativen Handlung, die in Wort, Bild, Ton oder Schrift transportierten Mitteilungen, Informationen oder Inhalte auffinden, wahrnehmen, verstehen, behalten und danach handeln können sollen (Maaß \& Rink, 2018, S. 24). Das Auffinden bezieht sich auf Informationen, die in erster Linie nur online abrufbar sind. Leichte Sprache, Vorlesefunktion oder modifizierbare Schriftgröße sind nützliche Hilfsmittel bei der - nachrichtentechnisch gesprochen - geräteunabhängigen Übertragung von codierten Informationen von der Quelle an die Perzepte und das zentrale sensorische System der*des Verbrauchenden. Der durch einen sinngebenden Reiz initiierte Wahrnehmungsprozess sollte nur so viele Ressourcen in Anspruch nehmen, dass das Verstehen der Mitteilungen im Anschluss ebenfalls möglich ist. Für das Präsentieren der Kommunikate ist eine syntaktisch klare und einfache Gestaltung erforderlich. Überdies sollten sie von der*dem Teilnehmenden im kognitiven Verarbeitungsprozess mit vorhandenen Wissensbeständen verknüpft werden können, um das Behalten zu erleichtern. Enthält die Information die Beschreibung einer optimalen Zielsituation, kann sie den Kommunikationsteilnehmenden eine Handlungsorientierung bieten (ebd. S. 25).

(Früh-)Förderung ist auf erlebbare Sinn- und Handlungserfahrungen in der Interaktion mit vertrauten Gegenständen und täglichen Routinen ausgerichtet. Technische wie nichttechnische Kommunikationshilfen können in gemeinsamer Gestaltung mit zentralen Bezugspersonen eingesetzt werden.

IN-books sind illustrierte Bücher, deren Textanteile normalerweise textgetreu in grafische Zeichen übersetzt werden und aus der Tradition der AAC/UK stammen. Das Ziel des Symboleinsatzes ist das Erhöhen von Lesbarkeit und Verständlichkeit, indem einzelne Begriffe oder Ausdrucksweisen angrenzend (generell unterhalb des Wortes) grafisch repräsentiert werden. Durch diese Anordnung kann eine positive Auswirkung auf die Aufmerksamkeitsspanne beim Lesen erzielt werden, was wiederum für das linguistische Verständnis der Kinder förderlich ist (vgl. Costantino, 2011). IN-books sind demnach unterstützende Werkzeuge für eine Kommunikation, bei der das Lesevergnügen im Zentrum steht. Das italienische Studienzentrum IN-book beschreibt das IN-book-Modell wie folgt (csinbook, 2017): 
- rispetto del mondo narrativo dell'autore, attraverso una traduzione simbolica fedele e precisa del testo originale

- rispetto del rapporto dialogico fra testo e immagine presenti nell'originale

- rappresentazione degli elementi linguistici in grado di caratterizzare gli aspetti specifici della lingua italiana, in particolare la morfologia

- organizzazione multimodale del simbolo associato all'etichetta alfabetica, con un marcatore di unità visiva (la riquadratura) che faciliti la scansione visiva e la lettura condivisa con modeling

- utilizzo della rappresentazione simbolica in versione monocromatica, per favorire i processi di automatizzazione della lettura

- utilizzo di sistemi simbolici in grado di supportare sia gli elementi astratti della lingua, sia gli elementi morfologici significativi

- cura nell'organizzazione della pagina, in termini di dimensione della simbologia, le distanze fra simboli, fra righe e fra paragrafi, la punteggiatura

- l'utilizzo delle immagini originali del libro

- la riduzione di testi di lunghezza e complessità maggiore pongono una sfida al modello. Valgono in ogni caso come elementi di riferimento, le caratteristiche sopra considerate ${ }^{3}$ (http://csinbook.altervista.org/il-modello-inbook/)

Beim Einsatz von IN-books ist auf die Gesamtsituation zu achten. Während des Vorlesens wird lautsprachlich der Leserhythmus eingehalten, während zugleich mit dem Finger auf den unteren Teil des umrahmten Symbols gezeigt wird: Die vorgelesenen Wörter werden durch das Medium der Sprache und die Symbole für das Kind verständlich. Costantino (2011) weist darauf hin, dass

3 Gefälligkeitsübersetzung:

- Respektierung der Erzählwelt des Autors durch eine getreue und präzise symbolische Übersetzung des Originaltextes;

- Respektierung der dialogischen Beziehung zwischen Text und Bild im Original;

- Darstellung der sprachlichen Elemente, die die spezifischen Aspekte der italienischen Sprache, insbesondere die Morphologie, charakterisieren;

- multimodale Organisation des Symbols, das mit dem alphabetischen Etikett verbunden ist, mit einer visuellen Einheitsmarkierung (das Quadrieren), die das visuelle Scannen und gemeinsame Lesen mit der Modellierung erleichtert;

- Verwendung der symbolischen Darstellung in einer monochromatischen Version, um die Prozesse der Automatisierung des Lesens zu erleichtern;

- Verwendung von Symbolsystemen, die sowohl die abstrakten Elemente der Sprache als auch die signifikanten morphologischen Elemente unterstützen;

- Sorgfalt bei der Organisation der Seite in Bezug auf die Größe der Symbole, die Abstände zwischen den Symbolen, zwischen den Zeilen und zwischen den Absätzen, die Interpunktion;

- Verwendung der Originalbilder aus dem Buch;

- das Reduzieren von längeren und komplexerenTexten stellt eine Herausforderung für das Modell dar.

- In jedem Fall gelten die oben betrachteten Merkmale als Referenzelemente. (http://csinbook. altervista.org/il-modello-inbook/ 01.09.2020) 
das Kind nie darum gebeten [wird], selber zu schauen oder zu zeigen. Es wird lediglich dem kontinuierlichen Zeigen ,ausgesetzt', Symbol für Symbol, spielerisch [...] durch das Modeling und ohne Notwendigkeit von Erklärungen oder Abrufen, lernt das Kind automatisch die Korrespondenz zwischen dem gehörten Wort, dem Symbol und dem geschriebenen Wort. (ebd., S. 222-223 - übersetzt von E.A. Emili)

Die gesprochene Sprache ist also für die Arbeit mit IN-books genauso zentral wie die Symbole in den Büchern selbst. Mitunter kann ein Kind durch das Mitführen seiner Hand den Leserhythmus besser erleben. Im Laufe der Zeit können unterschiedliche Situationen mit Abweichungen und verschiedenen Bezugspersonen durchgeführt werden, um eine vom Kind ausgehende Kommunikation zu fördern bzw. einen Wechsel in der Gesprächsführung zu ermöglichen (Wilken, 2002, S. 75, S. 81).

Neben schon vorgefertigten IN-books gibt es dank neuer Technologien und spezifischer Software (z. B. AraWord und Symwriter) die Möglichkeit, selbst IN-books herzustellen. Dadurch können Bücher, die von den Kindern im Kindergarten oder in der Familie genutzt und geliebt werden, zu personalisierten IN-books werden.

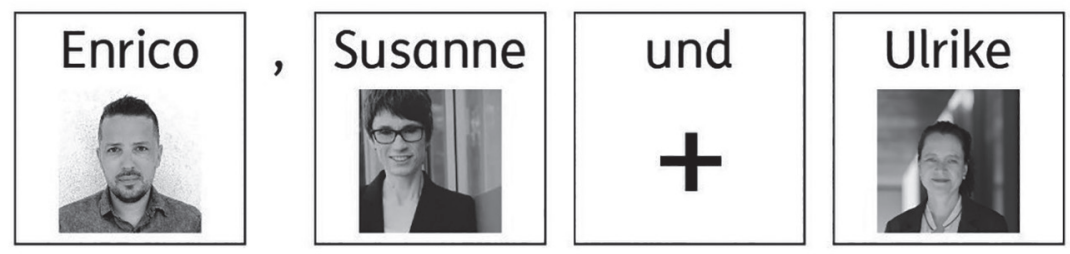

Widgit Symbols @ W Widgit Software 2002-2020 - www.widgit.com

Abb. 2: Personalisierte Symbole

Bei der Auswahl der Symbole ist darauf zu achten, dass die Qualität der gewählten Symbole so beschaffen ist, dass sie möglichst große situative Ähnlichkeit mit dem realen Sachverhalt aufweisen, kontrastreich aber dennoch detailarm sind überwiegend werden schwarz-weiße Symbole verwendet, nur die Farben selbst und die Figuren/Charaktere der Geschichte werden farbig abgebildet. So müssen nur wenige zusätzliche Codierungsvereinbarungen getroffen werden. Auch Nähe oder Abstand, Kontrast, Ähnlichkeit, Ausrichtung, Farbe und andere Gestaltungsfaktoren bieten Orientierung für das Erfassen der einzelnen Zeichen und ihrer Bedeutung. Der direkte Vergleich, da das Symbol unter den gedruckten Wörtern steht, erleichtert das Erfassen der Bedeutung der einzelnen Zeichen (Hallbauer \& Kritzinger, 2016, S. 6). 


\section{Das Projekt - Umwandlung von Bilderbüchern in Bücher mit Symbolen}

Augmentative and Alternative Communication (AAC) bzw. Unterstützte Kommunikation (UK) greifen u. a. auf technische Hilfsmittel zurück, um Kommunikationsund Sprachbarrieren zu reduzieren. Eine im italienischen Sprachraum bekannte Möglichkeit, Literatur für alle Kinder zugänglich zu machen, ist die Gestaltung von (Bilder-)Büchern, die den Text vollständig mit Symbolen unterlegen, sogenannte IN-books. In einer Reihe einschlägiger Studien wurden die positiven Auswirkungen auf Aufmerksamkeit und Sprachverständnis von Kindern durch die Verwendung von Symbolen beim Lesen dieser Bücher nachgewiesen (Cafiero, 2005; Binger \& Light, 2007; Raimondi, 2010; Costantino, 2011; Mirenda, 2014; Romski et al., 2015; ISS, 2015).

Allerdings ist deutschsprachige Kinderliteratur mit Symbolen in Buchhandlungen und Bibliotheken nicht zu finden. ${ }^{4}$ Dieses Defizit gab den Anlass zu einem Pilotprojekt ${ }^{5}$ in der EduSpace-Lernwerkstatt zur wissenschaftlichen Auswertung des Produktionsprozesses von Symbolbüchern sowie deren Anwendungsmodi aus den Perspektiven der Anwender*innen.

\subsection{Wissenschaftliche Projektbegleitung}

Das Forschungsinteresse liegt darin, die Perspektiven der Interaktionspartner*innen zu erheben und miteinander zu verschränken, um Struktur-, Prozess- und Ergebnisqualität der pädagogischen Handlungen verbessern zu können. Das Pilotprojekt wurde über einen Zeitraum von sechs Monaten angelegt. Die veränderte Situation aufgrund der COVID-19-Maßnahmen machte ein Abweichen vom ursprünglichen Erhebungsplan erforderlich, sodass die teilnehmende Beobachtung im Kindergarten zu einem späteren Zeitpunkt stattfinden muss. Unter weniger außergewöhnlichen Arbeitsbedingungen, als das gegenwärtig noch der Fall ist, können dann auch Aspekte der Sozialität in den Fokus gerückt werden, sodass ein Einblick zu Effekten in eher diskursiven Momenten des Einsatzes von IN-books und den darin rezipierten Normen möglich wird.

4 In Italien gibt es u. a. folgende Bücher mit Symbolen: Pinocchio, Der kleine Prinz, Der Zauberer von $\mathrm{Oz}$, Alice im Wunderland, Robbie regt sich auf, etc.

5 Forschungsprojekt von Enrico A. Emili (PI), in Zusammenarbeit mit Susanne Schumacher und unter der Leitung von Ulrike Stadler-Altmann (Wiss. Leitung EduSpace-Lernwerkstatt). 
Tab. 1: Projekt- und Forschungsablauf

\begin{tabular}{|c|c|}
\hline 022019 & $\begin{array}{l}\text { Projektstart/Workshop an der unibz } \\
\text { Einführung in die Thematik, Erläutern und Einüben der technischen } \\
\text { Handhabung der lizenzfreien Software mit limitierter Laufzeit, Erstellen } \\
\text { von Symboltafeln und Übertragen der Texte von ausgewählten deutsch- } \\
\text { sprachigen Bilderbüchern in die Symbolsprache }\end{array}$ \\
\hline 122019 & Technischer und methodischer Support durch die unibz \\
\hline $\begin{array}{l}122019 / \\
022020\end{array}$ & $\begin{array}{l}\text { Symbolbücher im Einsatz } \\
\text { Originalbuch und das in Symbole übersetzte Buch werden im KG zur } \\
\text { Verfügung gestellt. Beobachtung der Kinder mit Blick auf den konkre- } \\
\text { ten Umgang mit dem jeweiligen Exemplar als Gegenstand sowie in der } \\
\text { kommunikativen Interaktion. }\end{array}$ \\
\hline $04 / 052020$ & Leitfadeninterview \\
\hline $06 / 092020$ & $\begin{array}{l}\text { Datenverarbeitung } \\
\text { Transkription, Auswertung, Darstellung der Ergebnisse }\end{array}$ \\
\hline 102020 & $\begin{array}{l}\text { Projektende/Workshop } 2 \text { an der unibz } \\
\text { Rückmeldung der Ergebnisse }\end{array}$ \\
\hline
\end{tabular}

\subsection{Annahmen}

Bimodale, nicht-sprachliche Repräsentationsformen auf Basis von grafischen Zeichen stellen eine künstliche Kommunikationsform dar. Das bedeutet, dass beide Kommunikationspartner*innen - meist ein Kind und eine vorlesende Person-, die es nicht gewohnt sind, den Zugang zu Informationen durch Symbole zu nutzen, mehr oder weniger Zeit für die sprachlichen Übersetzungsleistungen brauchen. Sowohl in der bloßen Reproduktion von erlebter Anschauung als auch im Nachdenken über die Angemessenheit des symbolischen Ausdrucks liegt eine selbstständige Bewusstseinsleistung. Folglich müssen beide Interaktionspartner*innen in einer Kommunikationssituation bedenken, Was, Wann und Wie zu versprachlichen ist. Beispielsweise bietet das Programm Symwriter (mit Widgit-Symbolen) für jedes Wort mehrere Symbole an. Abstrakte Wörter, wie z. B. Liebe oder Fantasie sind schwieriger in grafische Zeichen umzusetzen, ebenso Artikel und Präpositionen (siehe Abb. 3). 


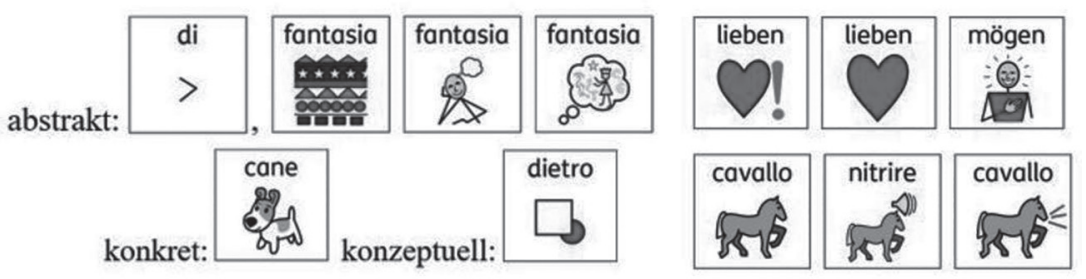

Widgit Symbols @ W Widgit Software 2002-2020 - www.widgit.com

Abb. 3: Beispiele für abstrakte und konkrete grafische Zeichen

In der Perspektive der pädagogischen Fachkräfte kann sich der Sachverhalt widerspiegeln als:

- Suchen, Finden und Verwerfen von Zeichen und Bedeutungen.

- Analysieren von fördernden und hemmenden Bedingungen bei der Herstellung.

- Benennen von Vorteilen/Hindernissen für die alltägliche pädagogische Arbeit. In der Perspektive der Kinder kann sich der Sachverhalt zeigen als:

- Suchen, Finden und Verwerfen von Zeichen und Bedeutungen.

- Erhöhte/verminderte Aufmerksamkeitsspanne beim Zuhören.

- Erhöhte/verminderte Aktivität mit Blick auf autonomes Lesen.

- Erhöhte/verminderte Aktivität mit Blick auf Peer-Interaktivität.

Mit dem hier vorgestellten Forschungsprojekt soll dieses Zusammenspiel zwischen der Expertise der pädagogischen Fachkraft und der Expertise des Kindes beleuchtet werden.

\subsection{Methoden}

Zum Einsatz kommen strukturierte Leitfaden-Interviews, da sich diese zur Erhebung von Daten besonders eignen, wenn einerseits alltägliches und wissenschaftliches Wissen zu rekonstruieren ist und dafür eine große Offenheit gewährleistet sein soll. Andererseits ist die Methode geeignet, den Erhebungsprozess durch die durch den*die Interviewende*n eingebrachten Themen zu strukturieren (Niebert \& Gropengießer, 2014, S. 121). Der Leitfaden folgt dem zeitlichen Ablauf des Gesamtprojekts. Er enthält fachsprachliche Wendungen, die in Alltagssprache paraphrasiert werden. Ziel des Interviews ist es, die fachlichen Wahrnehmungen, aber auch die sozialsystemischen Eindrücke der pädagogischen Fachkräfte zu rekonstruieren.

Ergänzend dazu sollen Beobachtungen der Vorlesesituation beim Einsatz der INbooks durchgeführt werden. 


\section{Leitfadeninterview}

Der Produktionsprozess der IN-books wird in den Interviews in den Mittelpunkt gestellt; somit sind die Erfahrungen und Erlebnisse der pädagogischen Fachkraft von Interesse. Spontane Interventionen wie Nachfragen zur Klärung des Gesagten oder andere Impulse sind möglich oder sogar willkommen, um das Fremdverstehen der Äußerungen sowie der skizzierten Situationen zu verbessern. Ein weiteres Mittel, um die Validität der Interviews sicherzustellen, ist die Verfahrens- und Datendokumentation durch Audiodateien in Kombination mit Protokollen. Folgende Leitfragen wurden verwendet:

1. Wie haben Sie von dem IN-Book Projekt erfahren?

2. Welche Arbeitsschritte waren für die Text-Übersetzung in Symbole notwendig? 3. Was war die größte Herausforderung/das größte Highlight?

4. Welche Erfahrungen konnten Sie mit dem Buch im Arbeitsalltag machen?

5. Welche weiteren Projekte können Sie sich vorstellen/würden Sie sich wünschen? Die Verarbeitung der Daten erfolgt anonym: Während der Transkription werden personenbezogene Angaben codiert und können der natürlichen Person nicht mehr zugeordnet werden.

Sample

Der Zugang zum Praxisfeld erfolgte über bestehende Kontakte in der EduSpaceLernwerkstatt. Interessierte pädagogische Fachkräfte aus dem Kindergarten mussten sich aktiv für eine Fortbildungsveranstaltung zum Thema „IN-books: Kinderbücher und Symbole“ anmelden. Zeitgleich erfolgte auch die Einwilligung zur Teilnahme am Forschungsprojekt. Insgesamt meldeten sich 14 pädagogische Fachkräfte aus mehreren Kindergärten eines Sprengels an.

\section{Datenauswertung: Qualitative Inhaltsanalyse}

Datengrundlage sind die Transkripte der Interviews. Für das hier zugrundeliegende Forschungsinteresse ist eine basale Stufe der Transkription angelehnt an Dresing und Pehl (2013) ausreichend. Kommentierungen und Erläuterungen in den Transkripten sind an Selting (2009) angelehnt.

In der Qualitativen Inhaltsanalyse nach Mayring (2000) kann das Datenmaterial induktiv, deduktiv oder induktiv-deduktiv ausgewertet werden. Für diese Studie wurden vordefinierte Kategorien methodisch abgesichert dem Text zugeordnet. Folgt man Nentwig-Gesemann (2011), Anders (2012) oder Fröhlich-Gildhoff et al. (2014), so besteht in Bezug auf Genese, Struktur und Auswirkungen professioneller Kompetenzen von frühpädagogischen Fachkräften ein Forschungsdefizit. Daher erscheint es sinnvoll, die Resultate der Lehrerforschung für die Primarschule und den Sekundarbereich in die Betrachtung von Kompetenzmodellen einzubeziehen. 
Tab. 2: Kompetenzkategorien für Lehrkräfte in Anlehnung an Baumert \& Kunter (2006)

\begin{tabular}{|c|c|c|}
\hline Kategorie & Definition & Ankerbeispiel \\
\hline Fachlich & $\begin{array}{l}\text { Domänenspezifisches Wissen + } \\
\text { praktischer Zugang zu diesem } \\
\text { Wissen }\end{array}$ & $\begin{array}{l}\text {... und gewisse Symbole ähneln } \\
\text { sehr der Unterstützten Kommu- } \\
\text { nikation. }\end{array}$ \\
\hline $\begin{array}{l}\text { Fachdidaktisch- } \\
\text { methodisch }\end{array}$ & $\begin{array}{l}\text { Zielgruppenspezifische Auswahl } \\
\text { von Aufgaben/ Formulieren } \\
\text { von Arbeitsaufträgen, mit } \\
\text { besonderem diagnostischem } \\
\text { Potenzial }\end{array}$ & $\begin{array}{l}\text { Ich habe mir das in meinen Au- } \\
\text { gen passendste Symbol ausgesucht } \\
\text { oder von dem ich denke, dass sich } \\
\text { Kinder das am besten merken } \\
\text { können. }\end{array}$ \\
\hline Pädagogisch & $\begin{array}{l}\text { Führung und Orchestrierung } \\
\text { von Lernprozessen, Wissen über } \\
\text { Entwicklung und Lernen, Di- } \\
\text { agnostik und Leistungsbeurtei- } \\
\text { lung sowie professionelles Ver- } \\
\text { halten im Kontext von Schule } \\
\text { und schulischer Umwelt }\end{array}$ & $\begin{array}{l}\text { Beim Wort "viele" gibt es mehrere } \\
\text { Symbole und eine Kindergärtne- } \\
\text { rin hat sich für ein anderes Sym- } \\
\text { bol entschieden und das irritiert } \\
\text { dann Kinder beim Lesenlernen } \\
\text { eher. }\end{array}$ \\
\hline $\begin{array}{l}\text { Affektiv-motiva- } \\
\text { tional }\end{array}$ & $\begin{array}{l}\text { Kontrollüberzeugungen und } \\
\text { Selbstwirksamkeitserwartun- } \\
\text { gen, Intrinsische motivationale } \\
\text { Orientierung: Lehrerendenen- } \\
\text { thusiasmus, Selbstregulation: } \\
\text { Engagement und Distanzie- } \\
\text { rungsfähigkeit }\end{array}$ & $\begin{array}{l}\text { Habe an dem Kurs teilgenommen } \\
\text { und es war sehr interessant. }\end{array}$ \\
\hline $\begin{array}{l}\text { Forschend-reflexi- } \\
\text { ver Habitus }\end{array}$ & $\begin{array}{l}\text { Hinterfragen der eigenen } \\
\text { Handlungen oder der Rolle } \\
\text { während des Augmentierens des } \\
\text { Bilderbuchs oder in der Inter- } \\
\text { aktion mit den Kindern oder } \\
\text { Kolleg*innen } \\
\text { Kritische Differenzierung der } \\
\text { eignen Aussagen. }\end{array}$ & $\begin{array}{l}\text { Das kann man nicht so nach- } \\
\text { vollziehen. Die Kinder schauen } \\
\text { ja mehr die Bilder an. Den Text } \\
\text { muss ich ja erst Mal vorlesen. Das } \\
\text { Verstehen sie ja noch nicht. }\end{array}$ \\
\hline
\end{tabular}

\subsection{Auswertung und Ergebnisse}

Die Datenlage ermöglicht eine zusammenfassende Betrachtung der fachlichen und sozialsystemischen Expertisen, die vor dem Hintergrund der Annahmen aufgezeigt und diskutiert werden. 


\section{Suchen, Finden und Verwerfen von Zeichen und Bedeutungen.}

Im ersten Workshop in der EduSpace-Lernwerkstatt im Rahmen des Forschungsprojekts wurden die pädagogischen Fachkräfte mit den Prinzipien von ACC/UK und IN-book vertraut gemacht. Anschließend wandelten sie selbst Kinderbücher in IN-Books um, d. h. ordneten den einzelnen Wörtern Symbole zu.

Da das Symwriter-Programm zur Erstellung der Symbole nicht auf Deutsch vorliegt und es keine vergleichbaren deutschsprachigen Programme gibt, mussten die pädagogischen Fachkräfte zuerst den Text des Kinderbuchs in das Italienische übersetzen. Erst danach konnten die entsprechenden Symbole ausgesucht werden. Das wurde als die größte Herausforderung angesehen, hier Beispiele aus den Transkripten:

(1) Das Programm bietet für einige Wörter verschiedene grafische Zeichen an. In diesem Fällen muss man das passende Symbol auswählen.

Bei Oscar liebt, es, heißt Oskar liebt <unk> und bei liebt waren zwei Symbole, dann war ein Herz und ein Herz mit Ausrufezeichen und <unk> [...] Und dann wusste ich nicht, was nehme ich jetzt für lieben und was nehme ich für mögen. Wo soll ich das Ausrufezeichen hinsetzen und wo nicht. (D12fünf10:152)

(2) Das Programm bietet auch keine präzisen Symbole für Geräusche und Laute von Lebewesen an.

Da kommt das Pferd und sagt Wiiieeehh zum Reiter zum Beispiel. Und dann bei dem willst $\mathrm{Du}$, also das Zeichen für willst fand ich irgendwie schwierig, weil eben willst $\mathrm{Du}$ ich dachte mir da das ist einfach für die Kinder schwierig darzustellen. [...] Und ich habe dann noch etwa hinzugefügt zum Beispiel bei dem Pferd, als es den Ton macht, da habe ich nochmal so ein paar Striche gemacht. (M06fünf14:61)

\section{Analysieren von fördernden und hemmenden Bedingungen bei der Herstellung.}

Der Umgang mit der kostenlosen Testversion der Software stellte eine von allen pädagogischen Fachkräften genannte Herausforderung dar.

Die Software runterladen war eine Herausforderung für mich. (M06fünf9:25)

Einige Bücher mussten im Vergleich zum Original vergrößert werden, um genügend Platz für die Symbole zu schaffen, damit die Kinder die Grafiken gut erkennen können

Also ich finde, dass Platz schon sehr wichtig ist. Also da würde ich sogar noch einen Platz dazu fügen oder irgendwie so probieren das es so geht, weil ich habe gemerkt, dass es ist wirklich wichtig, dass viel Platz und viel <unk>. Ja, dass die Symbole gut erkennbar sind. (D12fünf10:102) 


\section{Benennen von Vorteilen/Hindernissen für die alltägliche pädagogische Arbeit.}

Die widrigen Umstände (Lockdown ab Ende Februar 2020 in Südtirol) machten die Anwendung im Alltag und damit auch die Beobachtung nahezu unmöglich.

Dazu ist es leider nicht mehr gekommen. Dann wurde ja geschlossen. War ein bisschen schade. Aber das wäre ja für das nächste Jahr eine Idee. Dass man Inputs gibt und dann vertieft beobachten kann. Die Beobachtungszeit war zu kurz. (M22vier9:54)

Vorteilhaft erschien einigen pädagogischen Fachkräften der Einsatz von Symbolbüchern in Kindergärten, die überwiegend von Kindern mit Migrationshintergrund besucht werden.

Der Mehrwert in der pädagogischen Arbeit liegt sicher darin für Kinder, die wenig Sprache entwickelt haben oder für Kinder mit Migrationshintergrund, die eine andere Muttersprache haben, das unterstützend genommen werden kann. (M22vier9:52)

Also ich habe einfach gemerkt, gerade bei uns ist es einfach also, der Migra... Migrationshintergrund, der Anteil einfach sehr groß. Dass es gerade da sehr wichtig ist. [...] Und das ist mir nur so spontan gekommen, ich könnte das noch mal probieren. Wir machen immer mit der ganzen mit dem ganzen Kindergarten einen Singkreis. da sind wirklich alle 17 Kinder beieinander in der Turnhalle und ich hab' gemerkt, wie viele Kinder eigentlich nicht sehr interessiert sind, weil sie einfach auch nicht wissen, was wir gerade singen. (D12fünf10:340)

Der Einsatz als Springerin in unterschiedlichen Kindergärten des Sprengels wurde als einerseits ungünstig für die Beobachtung der Kinder im selbstbestimmten Umgang mit den Symbolbüchern empfunden. Andererseits konnten die pädagogischen Fachkräfte ihre Erfahrungen an andere Kolleg*innen weitergeben oder sich sogar austauschen.

Da war ich mal in einem anderen Kindergarten im Einsatz und habe in einer Bücherkiste gestöbert. Da ist mir ein Buch ins Auge gefallen auch mit Symbolen. Zunächst dachte ich, es sind dieselben Symbole. Aber da aus diesem Kindergarten keine Kollegin im Projekt war, dann habe ich nachgehakt. (D28vier15:39)

Die Begeisterung der pädagogischen Fachkräfte für ihre selbstgestalteten INbooks ist ein Faktor, der sich auf die Präsentation auswirkt und auf das Interesse der Kinder übertragen kann.

Mir ist vorgekommen, sobald ich es fertig gestellt habe, ich gebe es ihnen, weil ich hatte eine Freude, ihnen das zu zeigen. Und es ist auch sehr gut angekommen. (M06fünf9:144)

In der Ausschreibung der Fortbildung wurde darauf hingewiesen, Bücher mit wenig Text, einfachen Sätzen und Wiederholungen mitzubringen. Bei der Her- 


\section{Susanne Schumacher, Ulrike Stadler-Altmann und Enrico A. Emili}

stellung der Symbolbücher legten die pädagogischen Fachkräfte (mit einer Ausnahme) Wert auf die Objektähnlichkeit von Originalversion und Symbolversion mit Blick auf Farben, Formate und haptische Merkmale.

So hab' ich das auch immer genau dort platziert und war auch sehr froh, und habe es dann auch laminiert, also die einzelnen Blätter wollte ich innen es in Papier lassen, sodass es ähnlich ist wie im klassischen Buch. Nicht, dass es auch noch plastifiziert ist. Plastifiziert ist jetzt bei mir nur das Deckblatt. Das Innenleben ist nur Papier. (M06fünf9:57)

$\mathrm{Zu}$ den Bilderbüchern selber hätte ich schon was anzumerken. Dass sie nur fotokopiert und laminiert sind, spricht Kinder sehr wenig an. Das Haptische fehlt total. Wenn sie beide Varianten zur Verfügung haben, wählen Kinder sehr gern das Original, weil es einfach ein anderes Lesegefühl ist. (M29vier8:45)

Natürlich wird dieser Nachteil vermieden, sobald die Bücher in Italien verlegt werden. In der vorliegenden Studie ist nicht nur die fachliche, sondern auch die fachdidaktisch entsprechende Eigenproduktion der IN-books ein wesentlicher Punkt. Daher kann es für Kinder einer bestimmten Gruppe angemessen sein, die Größe des Buchs den Erfordernissen anzupassen.

Ich machte dieses Buch, also das bildunterstützte Buch, größer, also viel größer als das andere, weil die Umsetzung mit diesem, weil ich den Platz brauchte, auf den Text, dann machte ich dieses größer. Also es wurde ein schönes Buch. Vielleicht hat das auch dazu beigetragen, dass die Kinder dieses so gerne anschauten, weil es groß war. Jedenfalls größer als das andere. (D12fünf9:162)

Bei der Einführung der Symbolbücher als neues Literaturformat spielen Präsentationsreihenfolge und -häufigkeit sowie die Gruppengröße eine Rolle:

Man merkt dann auch bei den kleineren Kindern, die brauchen die Wiederholung stark. Und die haben so bestimmte Ideen und dann haben sie ein Lieblingsbuch, das lesen sie dann ein halbes Jahr. (M06fünf14:102)

Ich habe gesehen, dass die einschulenden Kinder dieses Buch gerne zur Hand genommen haben. Ich habe es ihnen nicht nur einmal vorgetragen, auch mehrere Male. Und ein paar Kinder haben dann angefangen, das Buch selbst in die Hand zu nehmen und Teilstücke hinaus zu lesen. (M06fünf11:82)

Grad'so in einer größeren in Gruppe ist immer schwierig, alle Details zu sehen, und ich hätte ihnen dann einfach das Buch zur Verfügung gestellt, an einem besonderen Platz. (D12fünf11)

Sie haben den Kindern das Buch vorgestellt in einer Art Schlusskreis. Sie waren sehr interessiert und aufmerksam. Die Kinder kannten das Buch eben überhaupt nicht. Und auch durch die Thematik sind die Kolleginnen sehr schnell in den Austausch mit den 
Kindern gekommen. Die Kinder haben auch gleich mitgedacht und mitgemacht, weil das Buch ja auch sehr anregt. (D28vier15:52)

Sind bereits Symbolbücher im Einsatz, muss auf die Gleichartigkeit der benutzten Software geachtet werden.

Das einzige Problem, das ich hatte, war, dass es dem Jungen zu viel wurde mit den Guckkarten, den METACOM-Symbolen, dann diese Symbole. Da habe ich noch nicht den richtigen Weg gefunden. Aber ich weiß, dass es unten im Kindergarten ganz viele Kinder gibt, die daran Interesse gehabt hätten. (M29vier17:52)

Den Angaben der pädagogischen Fachkräfte zufolge erwies sich der Mehrwert von IN-books in der Zusammenarbeit mit Kindern mit Beeinträchtigung nur teilweise.

Vielleicht kann ich die in einem Jahr beantworten. [...] Ob es für Kinder einen Mehrwert gibt, kann ich nicht sagen. Down Syndrom-Kind hat es nicht interessiert. Für die Gehörlosen war es zu verwirrend. (M29vier17:78)

Aufgrund der Kindergartenschließung war das in Eigenproduktion entstandene IN-book nur kurz im Einsatz und die pädagogischen Fachkräfte hätten sich mehr Beobachtungszeit gewünscht, allerdings sind auch nicht alle Kinder an Büchern interessiert.

Also mein Kind, mein Inklusionskind war total nicht interessiert an den Bildern. An den Bilderbüchern überhaupt nicht. Das hat ihn gar nicht interessiert. (D12fünf11:115)

[...] das andere Kind hat eine Beeinträchtigung, auch eine schwerere, sie war auch interessiert am Buch. (M06fünf910:25)

Einige Kinder, die das mit grafischen Zeichen erweiterte Bilderbuch gelesen hatten, haben es immer wieder in die Hand genommen und sich gegenseitig die Geschichte erzählt, und damit nach Einschätzung der pädagogischen Fachkraft ihren Wortschatz erweitert und ihre Formulierungsfähigkeit geübt.

Ich habe einfach gesehen, Sprache wird mehr gelebt. Also die Kinder haben mehr Möglichkeiten oder es bietet sich auch an, sich die Bücher selbst zu erzählen. Die Sprachentwicklung ist da wirklich gut behaftet. Ich würde wirklich gerne weitere Bücher übersetzen. (M06fünf9:114)

Ein Kind hat das Buch immer wieder zur Hand genommen und das Buch mit Freude selbst gelesen. Der Junge ist vier Jahre. Die Kollegin war sehr erstaunt, wie schnell die Kinder sich die Symbole merken. Logisch einige sind eindeutig, wie Kirschen. Aber auch andere Wörter, die man zumindest einmal gehört haben muss, daran erinnerten sich die Kinder ganz schnell. Das haben sie ganz schnell gespeichert. (D28vier15:59) 
Die Ergebnisse dieser ersten Forschungsphase geben auf Organisationsebene wichtige Hinweise für die Gestaltung des nächsten Forschungszyklus (siehe Tab. 3, Struktur- und Prozessqualität). Das Ziel des Projekts war die Einführung von Piktogrammen als Unterstützungsmedium für alle Kinder, um ihnen das Verständnis des Textes eines von pädagogischen Fachkräften vorgelesenen Bilderbuches zu erleichtern. Darüber sollten über Interviews die fachlichen Kenntnisse der pädagogischen Fachkräfte sowohl in der Anwendung mediengestützter Datenverarbeitung (siehe Tab. 3, Strukturqualität) als auch in der Aneignung und dem fachdidaktischen Einsatz einer Metasprache (siehe Tab. 3, Prozessqualität) ermittelt werden.

Darüber hinaus sollten die Erlebnisse und Erfahrungen bei der Gestaltung des Einsatzes der Bilderbücher mit Symbolen im Kindergartenalltag erfasst werden, um die Wirkung der Bücher mit Symbolen auf Kinder besser beschreiben zu können. Da dies nur in wenigen Kindergärten über einen Zeitraum von mehreren Tagen unmittelbar beobachtet werden konnte, sind die Aussagen in erster Linie Anhaltspunkte, die im nächsten Forschungszyklus systematischer dokumentiert werden müssen.

Mit der Schließung der Kindergärten wurde der Forschungsablauf unterbrochen, sodass die Beobachtungen nicht abgeschlossen sind und die Ergebnisse nur eine Anwender*innen-Perspektive abbilden. Gleichwohl können die Aussagen so verstanden werden, dass unterschiedliche Repräsentationsformen bereitgestellt wurden, die das Wahrnehmen und Erfassen von Informationen unterstützen (CAST, 2011).

In vielen Fällen erwies sich das Buch mit Symbolen insbesondere dann als erste Wahl, wenn die Geschichte neu und den Kindern unbekannt war. Ein Erklärungsansatz für diesen Sachverhalt könnte sein, dass die Kinder keine Vorlieben für die Originalversion entwickeln konnten. In einigen Interviews wurde betont, dass auf die Form und die Materialien des Buches zu achten sei, da mitunter die ästhetischen und ergonomischen Aspekte des Buches sogar den Inhalt einer Geschichte mehr oder weniger attraktiv machen können. In den Fällen, in denen die pädagogische Fachkraft selbst eine hohe affektiv-motivationale Grundhaltung zu Literatur im Allgemeinen und den Büchern mit Symbolen im Besonderen zeigte, übertrug sich die Begeisterung auf die Kinder. Sie wirkten interessierter und aufmerksamer beim Zuhören, vor allem Nicht-Muttersprachler oder Kinder mit Sprachschwierigkeiten.

In den Kindergärten, in denen das Buch mit Symbolen seit Januar im Einsatz war, kam es dazu, dass die Kinder gemeinsam mit der pädagogischen Fachkraft den Text lesen ${ }^{6}$ konnten. Einige von ihnen versuchten spontan, das Buch alleine zu lesen oder die Geschichte anderen Kinder nachzuerzählen, indem sie mit dem

6 Eher ein nachvollziehendes Mitlesen, da der Schriftspracherwerb erst im Kindergarten beginnt. 
Finger auf die Symbole zeigten. Ein Kind konnte das Geschehen sogar wortgetreu wiedergeben. Beide Sprachhandlungen sind Hinweise für ein gelungenes priming (siehe Tab. 3). Speziell das wechselseitige Erzählen und Weiterdenken einer Geschichte repräsentiert das grounding als gemeinsames Aushandeln eines sprachlichen Bedeutungsraumes (siehe Tab. 3). Kinder mit Behinderungen reagieren unterschiedlich auf Bücher mit Symbolen. Manche zeigen kein Interesse, andere wiederum schon. Somit bestätigt sich, dass die für das Autismus-Spektrum entwickelten Symbolbücher keine pauschale Unterstützung bieten. Es geht vielmehr darum, Kindern eine begleitende und vorübergehende Unterstützung (scaffolding) zu geben, die darauf abzielt, einen Text zu verstehen oder einfach eine andere Form des Lesens zu ermöglichen.

Tab. 3: Qualitätsebenen der Interventionen (adaptiert nach Giel 1999, S. 34)

\begin{tabular}{|c|c|c|c|}
\hline & Strukturqualität & Prozessqualität & Ergebnisqualität \\
\hline $\begin{array}{l}\text { EduSpace- } \\
\text { Lernwerk- } \\
\text { statt }\end{array}$ & $\begin{array}{l}\text { Konzeption einer } \\
\text { Lehr-Lernsituation } \\
\text { für erfahrene } \\
\text { Praktiker*innen }\end{array}$ & $\begin{array}{l}\text { Leiten einer Gruppe } \\
\text { mit heterogenen Vor- } \\
\text { kenntnissen, persönli- } \\
\text { ches Feedback, indivi- } \\
\text { dueller Support }\end{array}$ & $\begin{array}{l}\text { Qualitative Erkenntnisse } \\
\text { zum Zusammenspiel } \\
\text { verschiedener Repräsen- } \\
\text { tationssysteme aus unter- } \\
\text { schiedlichen Perspektiven }\end{array}$ \\
\hline \multirow{2}{*}{$\begin{array}{l}\text { Pädagogische } \\
\text { Fachkräfte }\end{array}$} & $\begin{array}{l}\text { Freiwilligkeit, } \\
\text { fachliches Inter- } \\
\text { esse, Perspektiv- } \\
\text { wechsel zum for- } \\
\text { schenden Lernen }\end{array}$ & $\begin{array}{l}\text { Impuls-Workshop, } \\
\text { eigenständiges Arbei- } \\
\text { ten, Beobachtung und } \\
\text { Dokumentation der } \\
\text { Effekte }\end{array}$ & $\begin{array}{l}\text { Reflexion der Arbeits- } \\
\text { schritte und Erfahrungen } \\
\text { mit Blick auf fachdidak- } \\
\text { tische sowie pädagogische } \\
\text { und affektiv-motivationa- } \\
\text { le Aspekte }\end{array}$ \\
\hline & $\begin{array}{l}\text { EDV-Grund- } \\
\text { kenntnisse, } \\
\text { lexikalisches, } \\
\text { semantische und } \\
\text { syntaktisches } \\
\text { Wissen }\end{array}$ & $\begin{array}{l}\text { Auswahl des Bilder- } \\
\text { buchs, Aneignen } \\
\text { einer Metasprache, } \\
\text { Übertragen von } \\
\text { Vokabeln/Grammatik } \\
\text { ins Deutsche und Itali- } \\
\text { enische }\end{array}$ & $\begin{array}{l}\text { Fachliche Kompetenzer- } \\
\text { weiterung: Metasprache } \\
\text { und einer multimodalen } \\
\text { Technik }\end{array}$ \\
\hline \multirow[b]{2}{*}{ Kinder } & $\begin{array}{l}\text { Interesse, Freiwil- } \\
\text { ligkeit }\end{array}$ & $\begin{array}{l}\text { Erhöhte Aufmerksam- } \\
\text { keit }\end{array}$ & $\begin{array}{l}\text { Erweiterung der Kommu- } \\
\text { nikationsfähigkeit }\end{array}$ \\
\hline & $\begin{array}{l}\text { Sprachliche Aus- } \\
\text { drucksfähigkeit, } \\
\text { Lesekompetenz }\end{array}$ & $\begin{array}{l}\text { Ko-Konstruktion mit } \\
\text { der pädagogischen } \\
\text { Fachkraft, mit anderen } \\
\text { Kindern }\end{array}$ & $\begin{array}{l}\text { Partizipation, Zugang } \\
\text { zu Sprache (priming \& } \\
\text { grounding) }\end{array}$ \\
\hline
\end{tabular}




\section{Das IN-book-Projekt als Teil der EduSpace-Lernwerkstatt}

Die Arbeit an der gemeinsamen Sache stellt im vorgestellten Projekt der EduSpaceLernwerkstatt die Brücke zwischen den beiden Referenzsystemen (Heid, 1995), der pädagogischen Praxis und der universitären Praxis in der EduSpace-Lernwerkstatt, dar. Dabei ist zu berücksichtigen, dass sich die universitäre Praxis einer Lernwerkstatt deutlich von der sie umgebenden universitären Praxis unterscheidet. Lernwerkstätten sind per se auf die pädagogische Praxis ausgerichtet und arbeiten mit einem handlungsorientierten Ansatz (Schudes, 2016; Coelen \& Müller-Naendrup, 2013). Allerdings werden Hochschullernwerkstätten von Vertreter*innen der pädagogischen Praxis als Teil einer fremden Universitätskultur wahrgenommen. Damit der Brückenschlag zwischen der EduSpace-Lernwerkstatt und der pädagogischen Praxis gelingt, sind neben konzeptionellen Entscheidungen und Weiterentwicklungen (siehe Stadler-Altmann, 2018, 2019; Stadler-Altmann et al., 2020) einzelne adressatenabgestimmte Projekte wie das hier vorgestellte nötig. Damit dieser und ähnliche Ansätze auch einen langfristigen, positiven Effekt für die Zusammenarbeit zwischen der EduSpace-Lernwerkstatt und Partner*innen aus der pädagogischen Praxis haben, müssen beide bereit sein, aufeinander zuzugehen und die jeweiligen vertrauten Arbeitsgebiete zu verlassen.

Zusätzlich benötigt eine Theorie-Praxis-Kooperation auf Augenhöhe Spielregeln (Hameyer, 2010) um langfristig erfolgreich zu sein. Aus Hameyers Überlegungen ist ein Quartett abzuleiten: Kohärenz-, Kompetenz-, Kontinuitäts- und Motivationsspielregeln.

Die Kohärenzregel verweist auf das Gesamtbild und die Ganzheit im kooperativen Theorie-Praxis-Transfer: Wie gut lässt sich die jeweilige Arbeit koordinieren und verzahnen? Im vorgestellten Projekt konnten die pädagogischen Fachkräfte ihre Arbeitsergebnisse aus dem Workshop in der EduSpace-Lernwerkstatt direkt in ihre pädagogische Arbeit einfließen lassen, da die eigenständig erarbeiteten Inhalte ihren professionellen Bedürfnissen entsprachen. Umgekehrt kann das Team der EduSpace-Lernwerkstatt den Workshop als Grundlage für ein Forschungsprojekt nutzen, das über den konkreten Inhalt und den Kontext des Südtiroler Kindergartens hinausweist. Die EduSpace-Lernwerkstatt dient hier als Rahmen für das Gesamtkunstwerk einer Kooperation auf Augenhöhe, die das gemeinsame Arbeiten und Lernen an einer Sache, dem IN-book und den dazugehörigen Kommunikationsprozessen, umfasst.

Die Kompetenzregel verweist auf den Anstieg von unterschiedlichsten Kompetenzen durch die Zusammenarbeit. Durch die gemeinsame Arbeit, wie z. B. im beschriebenen Projekt, können sich multiprofessionelle Teams entwickeln, die gegenseitig von ihren unterschiedlichen Erfahrungen, Herangehensweisen und Denkweisen profitieren. 
Die Kontinuitätsregel besagt, dass alle Anstrengungen nur Sinn ergeben, wenn es nicht bei einem einmaligen Austausch bleibt, sondern sich der Austausch verstetigen lässt. Deshalb bleibt es nicht bei einem einmaligen Workshop-Angebot: Die gemeinsame Arbeit an und mit IN-books wird mit weiteren Kooperationsprojekten mit weiteren Kindergartensprengeln in Südtirol fortgesetzt.

Die Motivationsregel hat innerhalb der Spielregeln eine Schlüsselfunktion, da eine auch langfristige Zusammenarbeit nur gelingt, wenn es immer wieder neue, interessante Aspekte in der Kooperation gibt. Motivation ist der Dreh- und Angelpunkt erfolgreicher pädagogischer Werkstattarbeit. Dies kann nur gelingen, wenn eine Lernwerkstatt, unabhängig davon, ob im Kindergarten, an einer Schule oder einer Hochschule, attraktive Angebote macht und auf Anfragen aus der pädagogischen Praxis und der Erziehungswissenschaft eingeht. Im Falle der EduSpace-Lernwerkstatt wird dies durch einen vielfältigen Austausch gepflegt: mit Kolleg*innen in der Fakultät für Bildungswissenschaften, in den Kindergärten und in den Schulen Südtirols, aber auch mit Studierenden und vermittelt durch Studierende, die ihre Erfahrungen aus den Praktika in die Lernwerkstatt einbringen können.

Das vorgestellte Forschungsprojekt ist also nicht nur ein Beispiel eines Professionalisierungsangebots für pädagogische Fachkräfte, sondern ebenso ein Beispiel für einen kooperativen Theorie-Praxis-Transfer. Die Anstrengungen in der EduSpaceLernwerkstatt gehen darüber hinaus. Ziel ist ein zirkulärer Transfer, in dem beide Partner sich in theoretischer und praktischer Weise anregen und herausfordern.

\section{Literatur}

Allgemeine Erklärung der Menschenrechte (AEMR). UN-Department for General Assembly and Conference Management German Translation Service, NY www.ohchr.org/EN/UDHR/Documents/UDHR_Translations/ger.pdf [zuletzt aufgerufen am 09.04.2020]

Anders, Y. (2012). Modelle professioneller Kompetenzen für frühpädagogische Fachkräfte. Aktueller Stand und ihr Bezug zur Professionalisierung. Expertise zum Gutachten „Professionalisierung in der Frühpädagogik“ im Auftrag des Aktionsrats Bildung. vbm.

Atagül, S. \& Müller, C. (2017). Transkulturelles Lernen mit mehrsprachigen Bilderbüchern. In S. Ozil, M. Hofmann, J.-P. Laut, Y. Dayioglu-Yücel, C. Zierau, \& K. Dickinson (Hg.), Türkischdeutsche Studien. Jahrbuch 2016. (139-159). Universitätsverlag.

Baumert, J. \& Kunter, M. (2006). Stichwort: Professionelle Kompetenz von Lehrkräften. Zeitschrift für Erziehungswissenschaft 9/4, 469-520

Binger, C. \& Light, J. (2007). The effect of aided AAC modeling on the expression of multi-symbol messages by preschoolers who use AAC, In Augmentative and Alternative Communication 23/1, $30-43$.

Bock, K. \& Griffin, Z. M. (2000). The persistence of structural priming: Transient activation or implicit learning? Journal of Experimental Psychology: General, 129(2), 177. https://doi.org/10.1037/00963445.129.2.177

Böhringer, J.; Bühler, P. \& Schlaich, P. (2011). Kompendium der Mediengestaltung. Konzeption und Gestaltung für Digital- und Printmedien. Springer-Verlag. DOI 10.1007/978-3-642-20587-3_5

Brandel, R.; Gottwald, M. \& Oehme, A. (Hg.) (2010). Bildungsgrenzen überschreiten: Zielgruppenorientiertes Übergangsmanagement in der Region. Wiesbaden: Springer. 


\section{Susanne Schumacher, Ulrike Stadler-Altmann und Enrico A. Emili}

Bubolz-Lutz, E. \& Mörchen, A. (2011). Die Lernwerkstatt - ein Entwicklungsarrangement für Erwachsenenbildner. In A. Mörchen, \& M. Tolksdorf (Hg.), Lernort Gemeinde. Ein neues Format der Erwachsenenbildung (103-138). wbv Media.

CAST (2011), Universal Design for Learning Guidelines version 2.0. Author.

Cafiero J.M. (2005), Comunicazione aumentativa e alternativa. Erickson

Coelen, H.\& Müller-Naendrup, B. (Hg.) (2013). Studieren in Lernwerkstätten. Springer

Costantino, M. A. (2011). Costruire libri e storie con la CAA. Gli IN-book per l'intervento precoce e l'inclusione. Erickson

Dresing, T. \& Pehl, T. (2013). Praxisbuch Interview, Transkription \& Analyse. Anleitungen und Regelsysteme für qualitativ Forschende (24-25). Zuletzt aufgerufen am 25.08.2020 unter www.audiotranskription.de/praxisbuch

Franzkowiak, Th. \& Frey, H. (1996). BLISS - Eine lebendige Sprache! In ISAAC Deutschland Gesellschaft für Unterstützte Kommunikation (Hg.), „Edi, mein Assistent “ und andere Beiträge zur Unterstützten Kommunikation. verlag selbstbestimmtes leben.

Fröhlich-Gildhoff, K., Weltzien, D., Kirstein, N., Pietsch, S. \& Rauh, K. (2014). Kompetenzen früh-I kindheitspädagogischer Fachkräfte im Spannungsfeld von normativen Vorgaben und Praxis. Expertise erstellt im Kontext der AG Fachkräftegewinnung für die Kindertagesbetreuung in Koordination des BMFSFJ. zuletzt aufgerufen am 09.02.2021 unter http://www.fruehe-chancen.de/informationen-fuer/zukuenftige-erzieherinnen-erzieher/studien-experteninterviews/expertise-zum-kompetenzprofil/

Glennen, S. L. (1997). Augmentative and Alternative Communication Assessment Strategies. In S.L.Glennen, \& D.C. DeCoste (Hg.), Handbook of Augmentative and Alternative Communication (149-192). Singular Publishing Group.

Gödert, W. (2018). Der konstruktivistische Ansatz für Kommunikation und Informationsverarbeitung, zuletzt aufgerufen am 07.09.2020 unter https://www.researchgate.net/publication/328415197_ Der_konstruktivistische_Ansatz_fur_Kommunikation_und_Informationsverarbeitung

Kindergarteninspektorat, Deutsches Bildungsressort (Hg.). (2008). Handreichungen zum Bildungsfeld Sprache, Schriftsprachkultur, Zwei-und Mehrsprachigkeit. Autonome Provinz Bozen

Hallbauer, A. \& Kritzinger, A. (2016). Vom Zeichen zum Symbol. Fachzeitschrift der Gesellschaft für Unterstützte Kommunikation (4).

Hameyer, U. (2010). Spielregeln in der Schulentwicklung, In E. Moning \& J. Petersen. (Hg.), Wandlungen komplexer Bildungssysteme. Festschrift für Jürgen Wiechmann. Bildung und Organisation 24 (93-108). Peter Lang.

Harnad, S. (1990). The Symbol Grounding Problem. Physica D 42, 335-346.

Heid, H. (1995). Zwischen Forschungspraxis und Praxisforschung. Über das mögliche Profil einer zukünftigen Modellversuchsforschung. In M. v. Twardy (Hg.), Modellversuchsforschung als Berufsbildungsforschung. Wirtschafts-, berufs- und sozialpädagogische Texte (295 -327). Botermann \& Botermann.

Huber, L. (2009). Warum Forschendes Lernen nötig und möglich ist. In L. Huber, J. Hellmer, \& F. Schneider (Hg.), Forschendes Lernen im Studium. Aktuelle Konzepte und Erfahrungen (9-35). UniversitätsVerlagWebler.

Istituto Superiore Sanità (2015), Il trattamento dei disturbi dello spettro autistico nei bambini e negli adolescenti. Color Art.

Keuneke, S. (2000). Geschlechtserwerb und Medienrezeption. Zur Rolle von Bilderbüchern im Prozess der frühen Geschlechtersozialisation. Leske + Budrich.

Knoblauch, H. (2017). Die kommunikative Konstruktion der Wirklichkeit, Neue Bibliothek der Sozialwissenschaften. Springer.

Kruger, D.; Parchmann, I. \& Schecker, H. (Hg.) (2014). Methoden in der 121 naturwissenschaftsdidaktischen Forschung. Springer-Verlag. 
Legislativdekret vom 24. Juli 1996, Nr. 434. Durchführungsbestimmungen zum Sonderstatut für die Region Trentino-Südtirol mit Änderungen und Ergänzungen zum Dekret des Präsidenten der Republik vom 10. Februar 1983, Nr. 89 betreffend die Schulordnung in der Provinz Bozen. Online unter: http://lexbrowser.provinz.bz.it/doc/de/dlgs-1996-434/legislativ-dekret_vom_24_ juli_1996_nr_434.aspx [letzter Aufruf: 02.01.2018].

Lohmar, D. (2008). Denken ohne Sprache? In F. Mattens (Hg.), Meaning and Language: Phenomenological Perspectives. Phaenomenologica 187 (169-194). Springer.

Maaß, C. \& Rink, I. (Hg.) (2019): Handbuch Barrierefreie Kommunikation. Kommunikation - Partizipation - Inklusion, Band 3. Frank \& Timme.

Mayring, P. (2000). Qualitative Inhaltsanalyse. Grundlagen und Techniken. Deutscher Studien Verlag.

Mirenda, P. (2014). Revisiting the mosaic of supports required for including people with severe intellectual or developmental disabilities in their communities. Augmentative and Alternative Communication (30), 19-27.

Müller, C. \& Schiefele, C. (2018). DaZ interaktiv und digital. Zur eigenständigen Produktion mehrsprachiger Bilderbücher mit Vorlesestiften. Fördermagazin Grundschule Deutsch (2), 21-25.

Müller, C. (2018). Literarische Bildung und mobiles Lernen in außerschulischen Erfahrungsräumen. bildung \& wissenschaft (3), 5-8.

Müller, C. (2016). Didaktische Potentiale digitaler Bilderbücher. Zur Förderung sprachlichen und literarischen Lernens mit digitalen Wimmelbüchern. In G. Scherer, \& S. Volz (Hg.), Im Bildungsfokus: Bilderbuchrezeptionsforschung. WVT, 353-370.

Nentwig-Gesemann, I., Fröhlich-Gildhoff, K., Harms, H. \& Richter, S. (2011). Professionelle Haltung - Identität der Fachkraft für die Arbeit mit Kindern in den ersten drei Lebensjahren. WiFF Expertisen Nr. 24. DJI.

Nieding, G. \& Ohler, P. (2008). Mediennutzung und Medienwirkung bei Kindern und Jugendlichen. In B. Batinic \& M. Appel (Hg.), Medienpsychologie (379-400). Springer.

Nußbeck, S. (2002). Das Konzept der Gestützten Kommunikation - Beschreibung und kritische Bewertung. In E. Wilken, (Hg.), Unterstützte Kommunikation. Eine Einführung in Theorie und Praxis (177-199). Kohlhammer.

Pickl, G. (1984). Eine Symbolsprache eröffnet nonverbalen Menschen die Welt der Kommunikation. In Der Sprachheilpädagoge (2), 77-81.

Prosetzky, I. (2002). Zur Bedeutung von Symbolen und deren Einsatz bei geistig behinderten Kindern ohne Lautsprache - Reflexionen aus linguistischer Sicht. IUnterstützte Kommunikation. ISAAC's Zeitung (1), 22-29.

Rahmenrichtlinien des Landes für die deutschsprachigen Kindergärten. Beschluss der Landesregierung vom 3. November 2008, Nr. 3990. Veröffentlicht im Beiblatt NR. 1 zum Amtsblatt der Region Trentino-Südtirol vom 25.Novemberr 2008, Nr. 48/i-ii.

Raimondi, F. (2010). Lettura e libro modificato. Università degli studi di Parma: tesi non pubblicata.

Romski, M., Sevcik; R.A., Barton-Hulsey, A. \& Whitmore, A.S. (2015). Early intervention and AAC: What a difference 30 years makes, In Augmentative and Alternative Communication 31/3, 181-202

Schnotz W. (2003). Bild- und Sprachverarbeitung aus psychologischer Sicht. In K. Sachs-Hombach (Hg.). Was ist Bildkompetenz? Bildwissenschaft (10) (25-42). Deutscher Universitätsverlag.

Schnotz, W. (2019). Pädagogische Psychologie Kompakt. Mit Online-Material. Beltz.

Schudes, S.; Bosse, D. \& Klusmeyer, J. (Hg.), Studienwerkstätten in der Lehrerbildung. Theoriebasierte Praxislernorte an der Hochschule (9-26). Springer VS.

Schumacher, S.; Stadler-Altmann, U. \& Riedmann, B. (2020). Verflechtungen von pädagogischer Theorie und Praxis. EduSpace Lernwerkstatt: stationär und mobil. In U. Stadler-Altmann, U., S. Schumacher, E. A. Emili, \& E. Dalla Torre (Hg.), Spielen, Lernen, Arbeiten in Lernwerkstätten. Facetten der Kooperation und Kollaboration (184-195). Klinkhardt. 
Schumacher, S.; Emili E. A. \& Stadler-Altmann, U. (2019). Effective Learning Experiences with UDL in teacher training at University. Richerche di Pedagogia e didattica. Journal of Theories and research in education 14/1, 165-191.

Schiefele, C. (2018). Formen und Möglichkeiten des Einsatzes digitaler Medien rund um Bilderbücher im inklusiven Deutschunterricht. URN:0111-pedocs-154330.

Selting, M. et al. (2009). Gesprächsanalytisches Transkriptionssystem 2 (GAT 2). Gesprächsforschung - Online-Zeitschrift zur verbalen Interaktion (10), 353-402.

Stadler-Altmann, U. (2019). EduSpace Lernwerkstatt als Verknüpfungsraum zwischen Praktikum und universitärer Lehre. In R. Baar, S. Trostmann \& A. Feindt (Hg.), Struktur und Handlung in Lernwerkstätten. Hochschuldidaktische Räume zwischen Einschränkung und Ermöglichung (201-213). Klinkhardt.

Stadler-Altmann, U. (2018). EduSpaces - Räume für kooperativen Theorie-Praxis-Transfer. Pädagogische Werkstattarbeit als Ansatz pädagogischer Professionalisierung. In M. Peschel \& M. Kelkel (Hg.), Fachlichkeit in Lernwerkstätten. Kind und Sache in Lernwerkstätten (227-245). Klinkhardt.

Stadler-Altmann, U.; Schumacher, S.; Emili, E. A. \& Dalla Torre, E. (2020), Hochschullernwerkstätten als Spielball der Bildungspolitik? Die EduSpace Lernwerkstatt in der Südtiroler Lehrerbildung zwischen nationalen und regionalen Bildungsinteressen. In: K. Kramer, D. Rumpf, M. Schöps, \& S. Winter (Hg.), Hochschullernwerkstätten - Elemente von Hochschulentwicklung? Ein Rückblick auf 15 Jahre Hochschullernwerkstatt in Halle und andernorts. Klinkhardt (im Druck).

Tetzchner von, S. \& Martinsen, H. (2000). Einführung in Unterstützte Kommunikation. Edition S.

Thole, W. (2013). Grundriss Soziale Arbeit. Ein einführendes Handbuch. VS Verlag für Sozialwissenschaften.

United Nations Department of Economic and Social Affairs Disability (2006). Convention on the Rights of Persons with Disabilities (CRPD). Zuletzt aufgerufen am 09.02.2021 unter https://www. un.org/development/desa/disabilities/convention-on-the-rights-of-persons-with-disabilities.html

Wedekind, H. (2013). Lernwerkstätten in Hochschulen - Orte für forschendes Lernen, die Theorie fragwürdig und Praxis erleb- und theoretisch hinterfragbar machen. In H. Coelen, \& B. MüllerNaendrup (Hg.), Studieren in Lernwerkstätten (21-28). Springer.

Wilken, E. (Hg.) (2002). Unterstützte Kommunikation. Eine Einführung in Theorie und Praxis. Verlag W. Kohlhammer. 